\title{
Performance Analysis of Channel Model CM1 \& CM2 of Wireless Body Area Networks
}

\author{
Sukhraj Kaur $^{1}$ and Dr. Jyoteesh Malhotra ${ }^{2}$ \\ ${ }^{1,2}$ ECE Department, GNDU RC Jalandhar, India \\ 'sukhrajkaur91@gmail.com, ${ }^{2}$ Jyoteesh@gmail.com
}

\begin{abstract}
The improvement in propagation characteristics of Wireless Body Area network is required to develop the high quality WBAN systems. In this paper, the power profile for WBAN channel model CM1 \&CM2 have been generated by using MICS band. The value of mean path loss has been calculated and compared for different channel models. Through extensive simulations, even the outage probability of different channel models has been plotted. The evaluation is done by calculating the value of Bit error rate for the given values of Signal to noise ratio using different modulation scheme.
\end{abstract}

Keywords: Wireless Body Area network(WBAN), Path Loss, channel model, Bit Error Rate(BER), Signal to Noise Ratio(SNR), Medical Implant Communication Service(MICS)

\section{Introduction}

Wireless Body Area network has great potential to revolutionize the future of medical technology. There is strong demand from medical and health care societies as well as information and communication technology (ICT) industries for introducing the WBAN technology. WBAN is the promising technology which use light weight, small size, ultra low power and intelligent sensors usually placed in(implanted) and around(wearable or placed closed to) the body. These sensors measure and process physiological signs of body and transmit them to the control unit (CU) without constraining the activities of the wearer. Doctors and caregivers can access the collected data for real-time diagnosis and trigger the treatment procedures in return. This increases the comfort level of the patient by freeing them from the need to be connected to hospital equipments that are required to monitor their conditions. This also prevents patient from any kind of sudden attack. WBANs could also play a huge role in military, competitive and non competitive athletics [2].

In 1996, Wireless body area network (WBAN) was first presented by T. G. Zimmerman. In April 2009, IEEE P802.15 working group for wireless Personal area networks (WPANs) has developed a channel model (IEEE 802.15.6) for WBAN which is for medical and non-medical devices that could be placed inside or on the surface of human body [3]. The fading power profile is generated in terms of received power with respect to transmit power, which is based on NICTA's (National Information and Communication Technology Australia) measurement results [2].

To design and develop a competent and reliable system suitable for WBAN, a knowledge of a radio propagation channel as well as a simple and generic channel model are inevitably required.

The organisation of paper is done as follow:

In the next section, brief description of WBAN channel model and terms related to it are given. Subsequently, the simulation methodology is followed by the result and discussions. The paper is finally concluded in the last section. 


\section{WBAN Channel Model}

In the WBAN systems, information propagates as electromagnetic waves from devices that are close to or inside the human body. The propagating wave experiences fading which is caused by energy absorption, reflection, diffraction and shadowing by body tissues and body posture. So, the human body is never an ideal medium for the propagation of radio waves. The multipath due to the environment around the body is also one of the factors causing fading which makes the channel models for WBAN different from the ones in the other environments.

The static path loss and impulse response models for the wearable and implantable WBAN includes miniature antennas are presented in [4]. These models are already contributed to IEEE 802.15.6. According to IEEE 802.15.6 document (Channel Model for Body Area Network), three types of nodes can be there:

1) Implant node: A node that is placed inside the human body. This could be immediately below the skin to further deeper inside the body tissue.

2) Body Surface node: A node that is placed on the surface of the human skin or at most 2 centimeters away.

3) External node (Gateway Node): A node that is not in contact with human skin (between a few centimeters and up to 5 meters away from the body).

A list of scenarios can be identified in which IEEE802.15.6 devices will be operating. These scenarios along with their description and frequency bands are listed in Table 1. The scenarios are determined based on the location of the communicating nodes (i.e., implant, body surface and external). The scenarios are grouped into classes that can be represented by the Channel Models (CM).

Table 1. List of Scenario and their Description [2]

\begin{tabular}{|c|l|l|c|}
\hline SCENARIO & DESCRIPTION & FREQUENCY BAND & CHANNEL MODELS \\
\hline S1 & Implant to Implant & $402-405 \mathrm{MHz}$ & CM1 \\
\hline S2 & Implant to Body surface & $402-405 \mathrm{MHz}$ & CM2 \\
\hline S3 & Implant to external & $402-405 \mathrm{MHz}$ & CM2 \\
\hline S4 & Body surface to Body & $13.5,50,400,600,900 \mathrm{MHz}$ & \\
& surface(LOS) & $2.4,3.1-10.6 \mathrm{GHz}$ & CM3 \\
\hline S5 & Body surface to Body & $13.5,50,400,600,900 \mathrm{MHz}$ & CM4 \\
& surface(NLOS) & $2.4,3.1-10.6 \mathrm{GHz}$ & \\
\hline S6 & Body surface to external & $900 \mathrm{MHz}$ & CM4 \\
& (LOS) & $2.4,3.1-10.6 \mathrm{GHz}$ & \\
\hline S7 & Body surface to external & $900 \mathrm{MHz}$ & $2.4,3.1-10.6 \mathrm{GHz}$ \\
& (NLOS) & & \\
\hline
\end{tabular}

This model is a statistical model and uses all the measurements carried out for CM1 and CM2 by NIST and NICT in [9]. This paper uses the fading power profile of WBAN channel which includes fading and path losses [2].

\subsection{Fading}

In the WBAN communications, propagation paths can experience fading due to different reasons, such as energy absorption, reflection, diffraction, shadowing by body, and body posture and the multipath signal due to the environment around the body. Fading can be categorized into two categories

- $\quad$ small scale fading

- $\quad$ large scale fading 
Small scale fading is caused by rapid fluctuations in the amplitude and phase of the received signal within a small local area in small period of time due to small changes in location of the on-body device or body positions. It occurs due to variation in the relative position between transmitter and receiver [2].

Large scale fading refers to the signal attenuation due to mobility over larger areas. It occurs due to variation in distance covered by signal between antenna positioned on the body and external node (home, office, etc.,) because of diffraction from large surrounding objects.

\subsection{Path Loss}

In WBAN, path loss is both distance and frequency dependent. The path loss model between the transmitting and the receiving antennas can be represented as a function of the distance (d).Based on the Friis formula [1] in free space path loss in $\mathrm{dB}$ is expressed by (1) as follows

$$
P L(d)=P L_{o}+10 n \log \left(\frac{d}{d_{o}}\right)
$$

Where $\mathrm{PL}_{\mathrm{o}}$ is the path loss at a reference distance $\mathrm{d}_{\mathrm{o}}$, and $\mathrm{n}$ is the path - loss exponent.

\subsection{Shadowing}

Shadowing is the process of blocking of signal by large objects which are encountered due to the variation in the environment surrounding the body or even due to movement of the body parts. It causes variation in path loss of signal from that of mean value for a given distance as shown in (1). When considering shadowing, the total path loss i.e., PL can be expressed by (2) [2]

$$
P L=P L_{o}+S
$$

Combining (1) and (2), total path loss is

$$
P L=P L_{o}+10 n \log \left(\frac{d}{d_{o}}\right)+S
$$

\subsection{Outage Probability (OP)}

The OP is a standard performance measure of the quality of the transmission operating over fading channels. The OP can be given as the probability that the receiver performs poorly than a specified threshold BER. The signal outage probability is defined as the probability that the instantaneous SNR falls below a certain threshold, $\gamma_{t h}$ i.e., [5]

$$
P_{\text {out }}\left(\gamma_{t h}\right)=P\left(S N R<\gamma_{t h}\right)
$$

\subsection{Bit Error Rate}

The bit error rate is the ratio of number of error bits to the total transferred bits during a time interval for which the performance is measured. It is also called bit error ratio (BER).The probability of bit error rate is an approximate estimation of bit error rate and is calculated as follows:

2.5.1. M-ary PAM: The probability of symbol error is

$$
P_{M}=\frac{1}{\sqrt{2 \pi}} \int_{-\infty}^{\infty}\left[1-\left(\frac{1}{\sqrt{2} \pi} \int_{-\infty}^{y} e^{-x^{2} / 2} d x\right)^{M-1}\right] \exp \left[-\frac{1}{2}\left(y-\sqrt{\frac{2 E_{S}}{N_{0}}}\right)^{2}\right] d y
$$


Where $\frac{E_{b}}{N_{o}} \rightarrow$ signal to noise ratio

The average bit probability error is

$$
P_{m}=\frac{P_{M}}{M-1}=\frac{P_{M}}{2^{k}-1}
$$

2.5.2. M-ary BOK: The probability of symbol error is

$$
P_{M}=1-\frac{1}{\sqrt{2 \pi}} \int_{-\infty}^{\infty}\left(\frac{1}{\sqrt{2 \pi}} \int_{-\infty}^{v+\sqrt{\frac{2 \gamma M}{M-1}}} \exp \left(\frac{-x^{2}}{2}\right)\right)^{M-1} d x * \exp \left(-\frac{v^{2}}{2}\right) d v
$$

where $\gamma=\gamma_{\mathrm{b}} \log (M) / \log (2)$ is the SNR per symbol and $\gamma_{\mathrm{b}}$ is the received SNR per information bit.

The average bit probability error is

$$
P_{m}=P_{M} \frac{M}{2(M-1)}
$$

\section{Simulation Methodology}

Simulation is the process of designing a model of a real system and conducting experiments with this model for the purpose of understanding the behavior of the system and for evaluating various parameters for the operation of the system. However it is not possible to model every parameter of human body channel through commonly used formulas in other wireless channels because of its complex tissue structure but major parameters have been taken into considerations. Also measurement of various human body parameters is not an easy task. So it is necessary to design channel model using standard measurements and in this channel model all measurements, i.e., sample rate, spreading bandwidth etc. are taken from NICTA channel measurements. Since the performance analysis of the WBAN receiver is based on statistical model of the channel [7]. The simulation model is used here to statistically generate the fading power profile of WBAN channel [3]. In order to generate an initial signal, that matches the desired fading statistics using order statistics, the following steps are being used.

Step1:Generate Path_Loss[] = Implant_PL_Model(d,N_point, model_id) , of CM1 \&CM2.

Step2:Generate power profile ([signal,time_samp, mean_path_loss] = generate_power_profile_wmban(), of Human BAN channel .

Step3: Calculate absolute value of signal.

Step4: Calculate received SNR of each sample of power profile with the help of transmitted SNR and mean_path_loss .

Step5: Calculate received signal to noise ratio.

Step6: Calculate BER for PAM and M-ary BOK receiver.

Step7:Calculate Outage Probability of CM1 \& CM2 channel model.

Step8:Outage Probability vs normalized threshold power graphs are plotted.

Step9: BER vs SNR curves are plotted . 


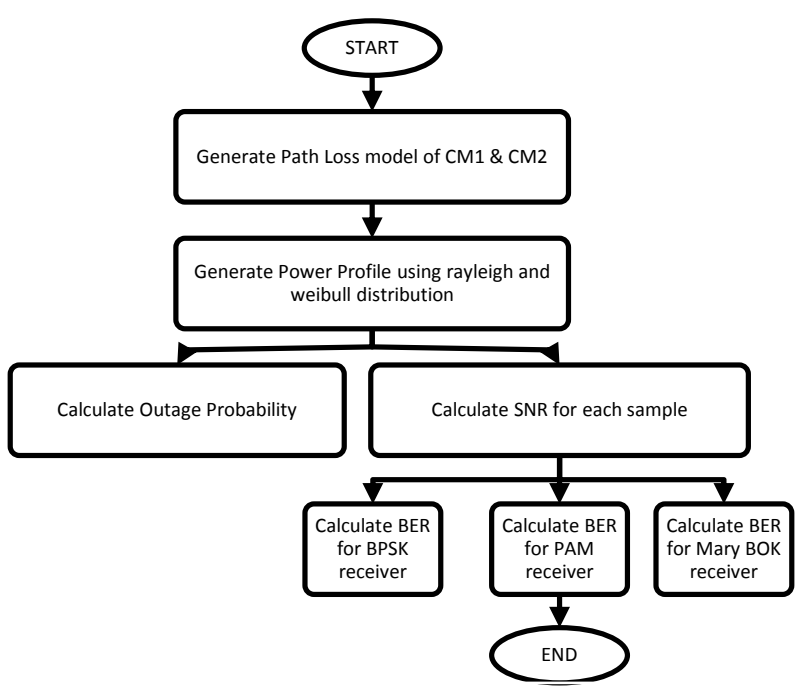

Figure 1. Flow Chart for Generation of SNR vs BER and Outage Probability

\section{Result and Discussion}

In this section, the performance of channel models (CM1 \& CM2) of wireless body area network is presented in terms of fading profile, outage probability and BER using MICS band of frequency ranging from $402 \mathrm{MHz}$ to $405 \mathrm{MHz}$.

\subsection{Fading Power Profile}

Extensive simulations have been carried out in this work to observe the variations of the channel fading in terms of carrier frequency within the constraints specified by NIST and NICT in [6]. From the description of the simulation model given in the previous section, fading power profile of WBAN channel for different values of carrier frequency for CM1 and CM2 have been plotted in Figure 1. Channel gain represents the gain in the power of the signal while transferring the data from transmitter end to receiver end. As the value of the channel gain is always negative in the graphs, it represent that there is loss in power of signal while travelling of signal from transmitter to receiver i.e. there is fading of the signal.

\subsubsection{Deep implant to on-body:}
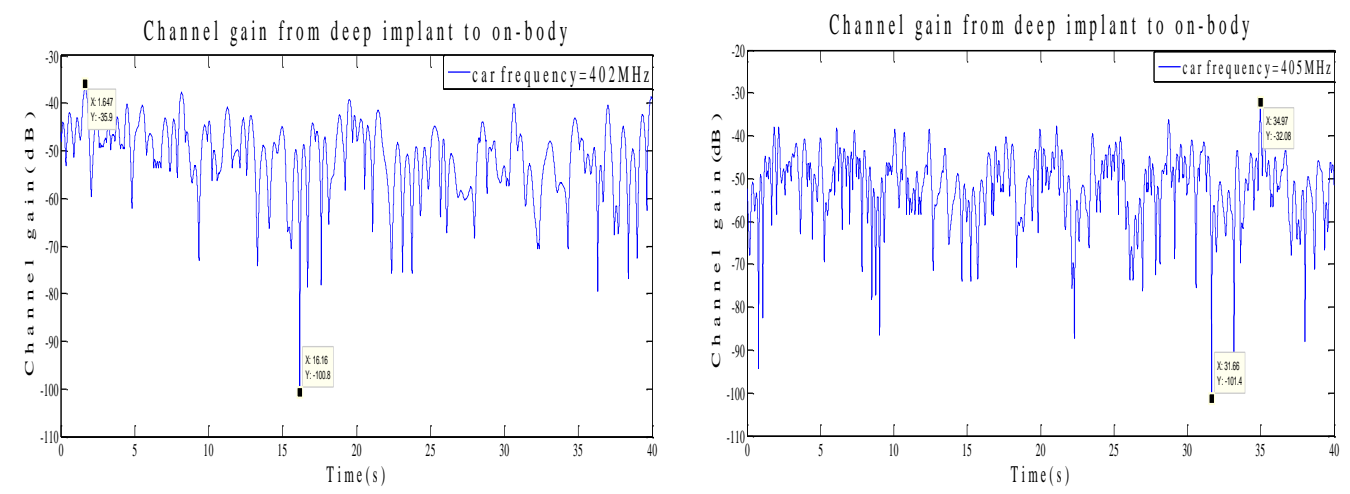

Figure 2(a). Fading in Channel Deep Implant to on -body for Different Carrier Frequency 
With increase in frequency from $402 \mathrm{MHz}$ to $405 \mathrm{MHz}$ the fading level of signal travelling from deep implant to on-body goes up.

\subsubsection{Near Surface implant to on-Body:}
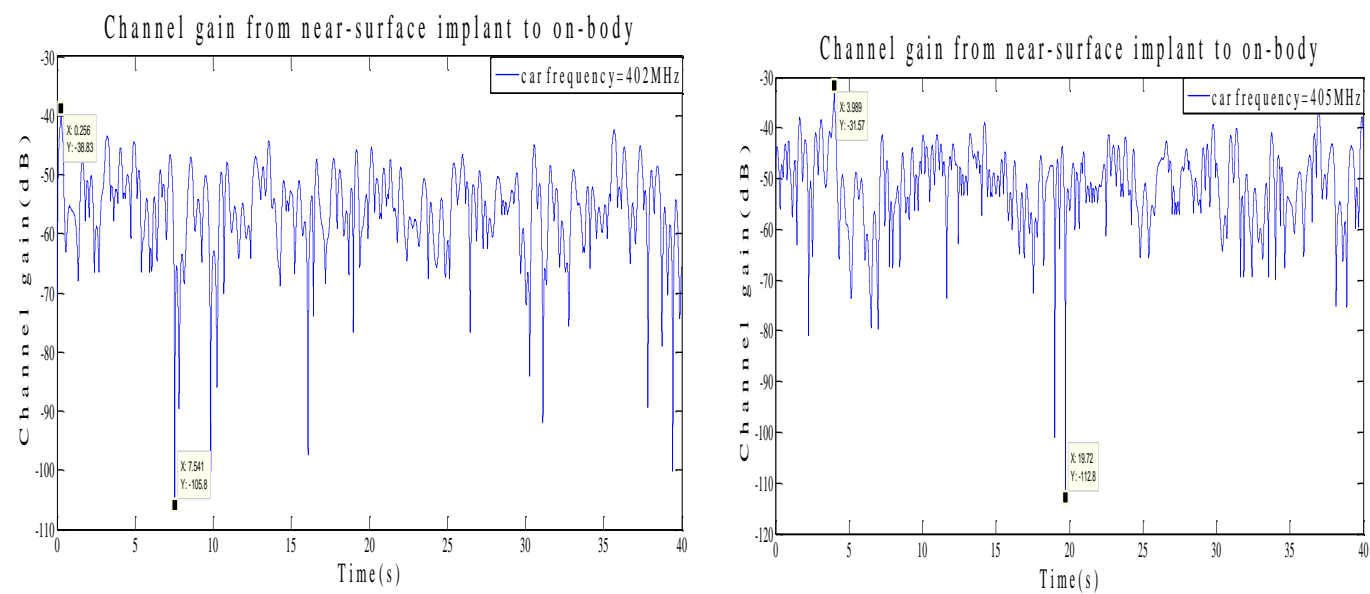

Figure 2(b). Fading in Channel Near-Surface Implant to on -body for Different Carrier Frequency

The propagation of signal from near-surface implant to on-body node faces more fading when there is increase in frequency from $402 \mathrm{MHz}$ to $405 \mathrm{MHz}$.

\subsubsection{Deep Implant to Implant:}
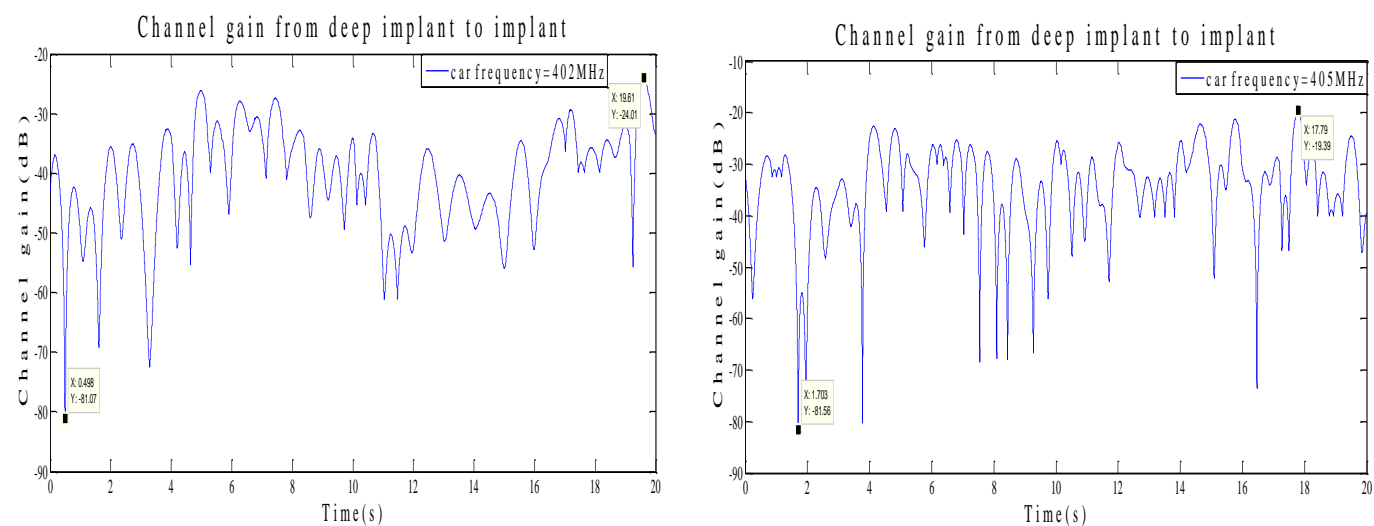

Figure 2(c). Fading in Channel Deep Implant to Implant for Different Carrier Frequency

In deep implant to implant communication, the fading of the signal rises up with increase in frequency from the $420 \mathrm{MHz}$ to $405 \mathrm{MHz}$. 


\subsubsection{Near-Surface Implant to Implant:}
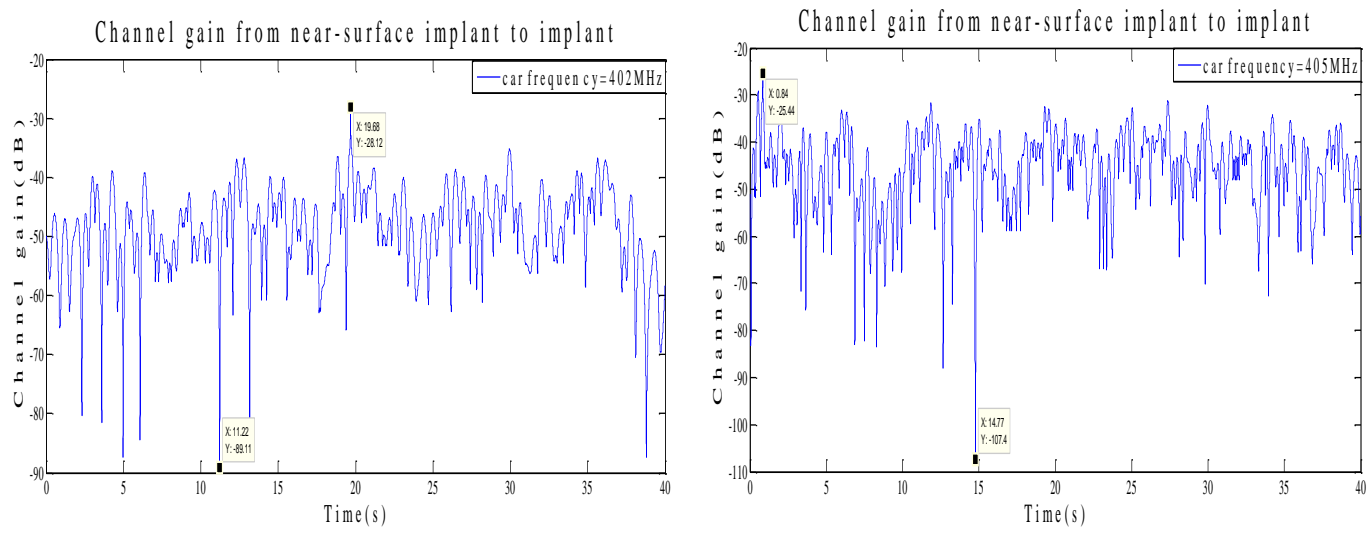

Figure 2(d). Fading in Channel Near-Surface Implant to Implant for Different Carrier Frequency

In case of near-surface implant to implant channel model, the increase in frequency from $402 \mathrm{MHz}$ to $405 \mathrm{MHz}$ cause the corresponding rise in the fading level of the signal.

For calculation of mean fading in Table 2 , the values of velocity is $1.5+4 *$ rand (rand function generate values from 0 to 1.), number of scatterers are 100 and sample rate is kept at $1 \mathrm{kHz}$.

Table 2. Mean Fading of Different Channel Models (CM1 \& CM2)

\begin{tabular}{|l|c|c|}
\hline \multirow{2}{*}{\multicolumn{1}{|c|}{$\begin{array}{c}\text { CHANNEL } \\
\text { MODEL }\end{array}$}} & \multicolumn{2}{c|}{ FREQUENCY } \\
\cline { 2 - 3 } & $402 \mathrm{MHz}$ & $405 \mathrm{MHz}$ \\
\hline Deep implant to on-body & 64.9 & 69.32 \\
\hline Near-surface implant to on-body & 66.97 & 81.23 \\
\hline Deep implant to implant & 57.06 & 62.17 \\
\hline Near-surface implant to implant & 60.99 & 81.96 \\
\hline
\end{tabular}

As the value of carrier frequency increases, the amount of fading present in the channel also increased which is clear from the Figure 2. From the Table 2, it is clear that the amount of fading is lower at $402 \mathrm{MHz}$ as compares to the $405 \mathrm{MHz}$ of carrier frequency.

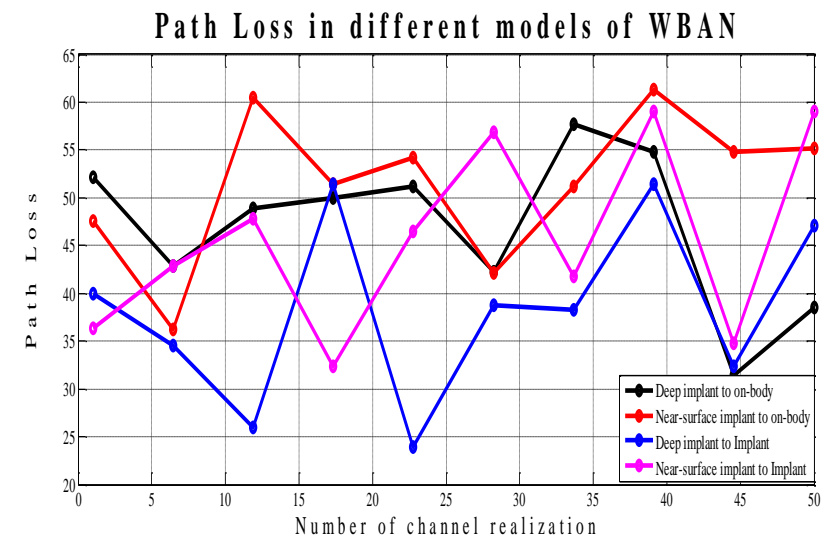

Figure 3. Path Loss of CM1 \& CM2 of WBAN 
From the above graph (Figure 3), it is clear that performance of the channel deep implant to implant is better than other channel, while the near-surface implant to on-body shows the poorest results.

\subsection{Outage Probability}

In this section, the outage probability of channel model CM1 \& CM2 are compared with respect to each other. Even the outage probability of channel models CM1 \& CM2 of WBAN have been plotted for varying distance between transmitter and receiver.

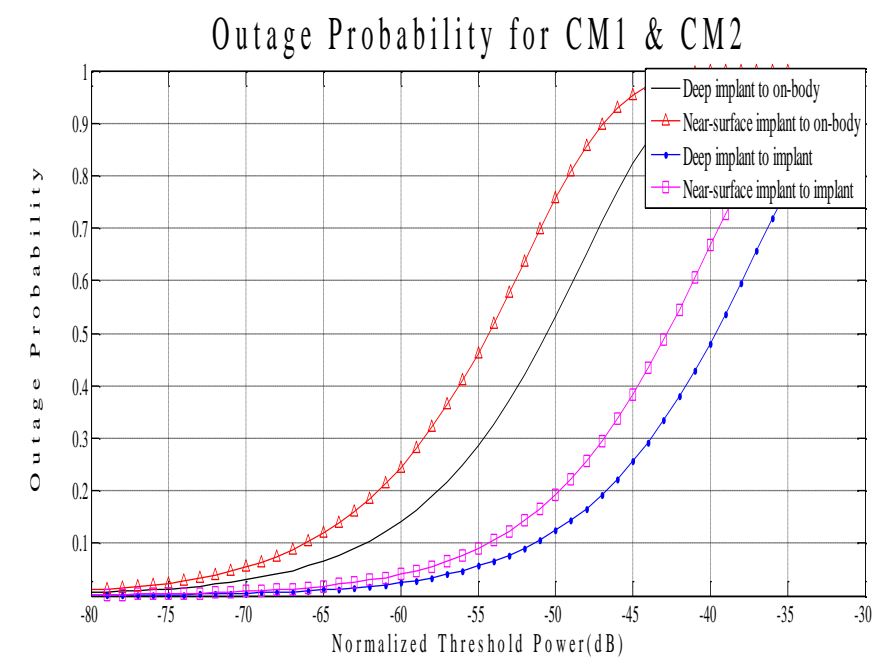

Figure 4. Outage Probability of Different Channel Models (CM1 \& CM2)

The outage probability of channel models CM1 \& CM2 in Figure 4, shows that the performance of deep implant to implant is better than another scenarios of CM1 \& CM2.

Table 3. Outage Probability of Different Channel Model at Different Normalize Threshold Power

\begin{tabular}{|l|c|c|}
\hline \multirow{2}{*}{$\begin{array}{c}\text { CHANNEL } \\
\text { MODELS }\end{array}$} & \multicolumn{2}{|c|}{\begin{tabular}{c} 
NORMALIZED THRESHOLD POWER (dB) AT \\
\cline { 2 - 3 }
\end{tabular}} \\
\cline { 2 - 3 } & $10 \%$ & $90 \%$ \\
\hline Deep implant to on-body & -62 & -43 \\
\hline Near-surface implant to on-body & -66 & -47 \\
\hline Deep implant to implant & -51 & -32 \\
\hline Near-surface implant to implant & -54 & -35 \\
\hline
\end{tabular}


Outage Probability for Deep implant to on-Body

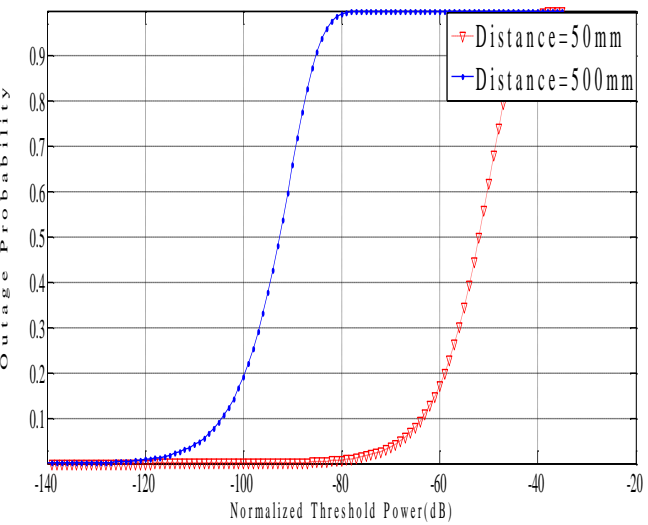

Outage Probability Deep implant to implant

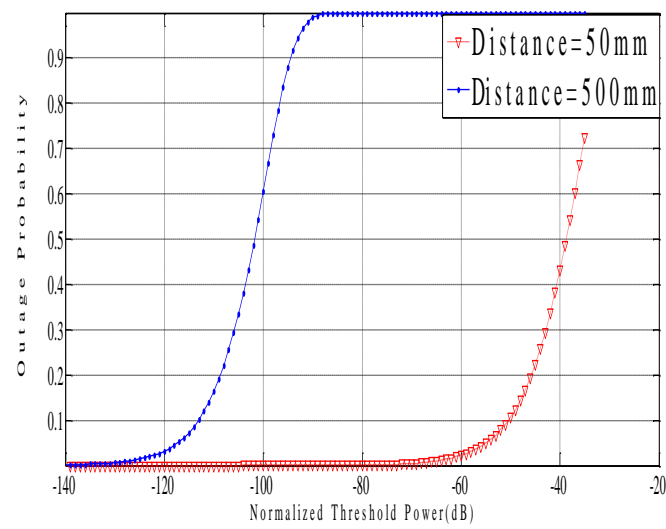

Outage Probability for Near-surface implant to 0n-body

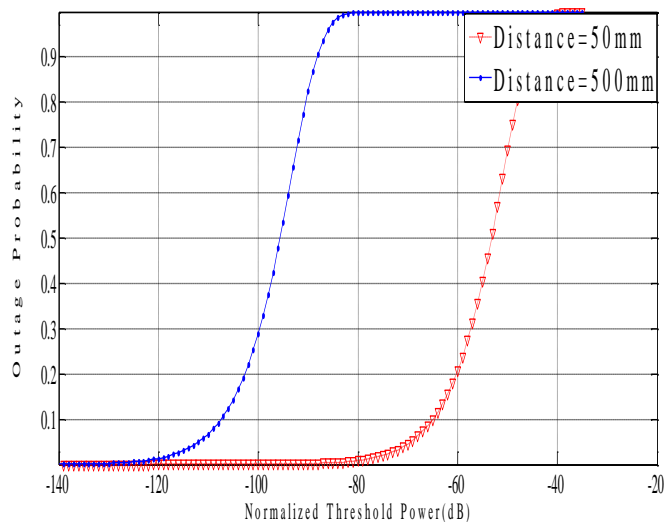

Outage Probability for Near-surface implant to implant

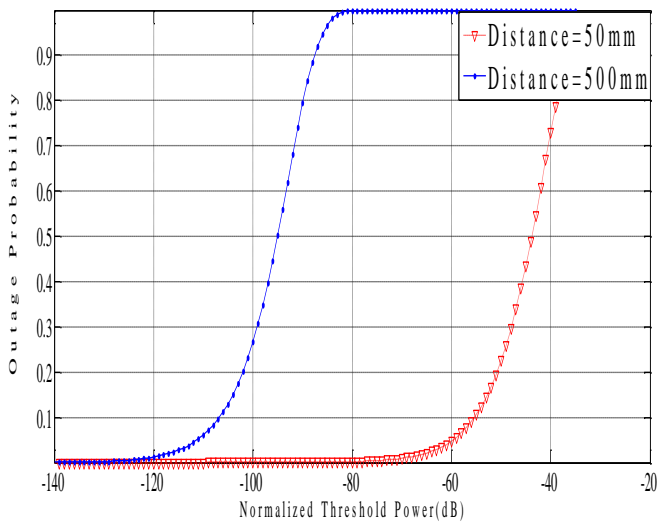

Figure 5. Outage Probability of Channel Model CM1 \& CM2 at Different Distances $(50 \mathrm{~mm}$ and $500 \mathrm{~mm})$

From Figure 5, it is clear that performance of different channel models degrades with increase in distances from $50 \mathrm{~mm}$ to $500 \mathrm{~mm}$. The more far away are the transmitter and receiver the more degradation is in the performance of the channel.

Table 4. Normalized Threshold Power of Different Channel Models at Different Distances

\begin{tabular}{|l|c|c|c|c|}
\hline \multirow{2}{*}{ CHANNEL MODEL } & \multicolumn{4}{|c|}{ NORMALIZE THRESHOLD POWER (dB) AT OUTAGE } \\
& \multicolumn{3}{|c|}{$10 \%$} & $50 \%$ \\
\cline { 2 - 5 } & $50 \mathrm{~mm}$ & $500 \mathrm{~mm}$ & $50 \mathrm{~mm}$ & $500 \mathrm{~mm}$ \\
\cline { 2 - 5 } & -64 & -104 & -44.5 & -85 \\
\hline Deep implant to on-body & -65 & -107 & -46 & -88 \\
\hline $\begin{array}{l}\text { Near-surface implant to on- } \\
\text { body }\end{array}$ & -51 & -113 & -29 & -94 \\
\hline Deep implant to implant & -55 & -107 & -36 & -88 \\
\hline $\begin{array}{l}\text { Near-surface implant to } \\
\text { implant }\end{array}$ & & & & \\
\hline
\end{tabular}


In the above table 4 , the rise in the outage probability is observed with increase in distance from $50 \mathrm{~m}$ to $500 \mathrm{~mm}$.

\subsection{Bit Error Rate}

Bit Error Rate (BER) is the number of bits of error occurs within one second in the transmitted signal. Extensive simulations have been carried out in this work to observe the variations of the BER performance for PAM and BOK modulation [3] within the constraints specified by NICTA. In this graphs, $\mathrm{y}$-axis represents bit error rate and $\mathrm{x}$-axis represents signal to noise ratio (SNR). BER vs. SNR has been plotted for M-PAM and MBOK modulation scheme as follow:

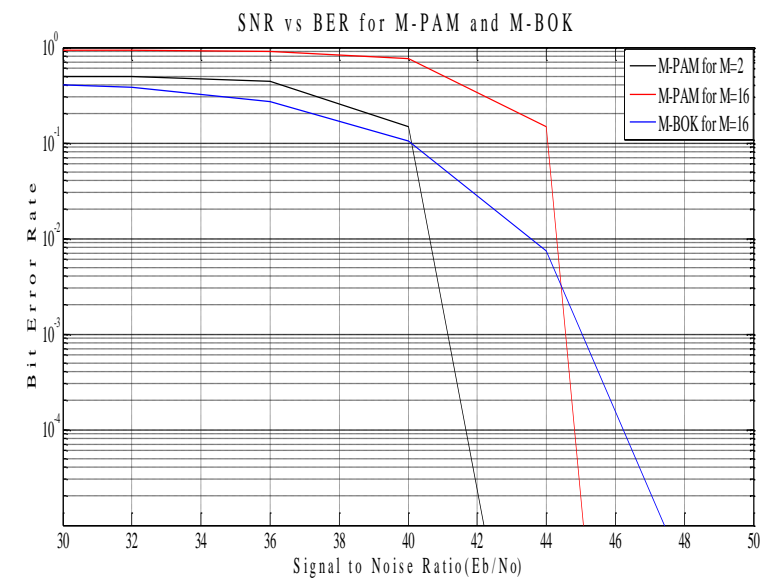

Figure 6. BER vs. SNR for M-PAM and M-BOK

\section{Table 5. Comparison of BER for Different Modulation}

\begin{tabular}{|l|c|c|}
\hline \multirow{2}{*}{ S. no. } & S/N ratio(x) & Bit error rate \\
\hline \multirow{2}{*}{ 2-PAM } & 40 & .149 \\
\cline { 2 - 3 } & 44 & $3.9 \mathrm{e}-009$ \\
\hline \multirow{2}{*}{ 16-PAM } & 40 & .77 \\
\cline { 2 - 3 } & 44 & .1455 \\
\cline { 2 - 3 } & 40 & .105 \\
\hline
\end{tabular}

From Figure 6, it is clear that performance of 2-PAM is always better than that of 16PAM. Even the results of 2-PAM is far better than BOK.

\section{Conclusion}

WBAN seems to be very prominent technology in the near future and various applications are being designed based on this technology in the health care sector. However there are various design challenges ahead of communication engineers to enable the WBAN based applications a reality. So in this work, extensive simulations have been carried out considering the different channel conditions of different channel models of WBAN. The results obtained in this paper shows that amount of fading is minimum in case of deep implant to implant channel. Even the increase in frequency or distance increases the fading of the corresponding channel. Even the amount of error in case of PAM modulation scheme is far less than Book's PAM modulation scheme at $402 \mathrm{MHz}$ of 
frequency and less distance provides more effective channel. Thus, the results obtained in this paper will be of great help in facilitating the network designers for systematic planning of the WBAN based products in the future.

\section{Acknowledgment}

We owe our debt of gratitude to our department for the vision which inspired us to conceive this work. We thank almighty and our parents without whom this project would not be possible.

\section{References}

[1] H. Bai and M. Atiquzzaman, "Error modelling schemes for fading channels in wireless communication: A survey", IEEE Communications Surveys and Tutorials, vol. 5, (2003) December, pp. 2-9.

[2] K. Y. Yazdandoost and K. Sayrafian-Pour, "Channel Model for Body Area Network (BAN)", IEEE802.15.6 technical contribution, document ID: 15-08-0780-09-0006, (2009) April 27, pp. 41-56.

[3] H.-B. Li and R. Kohno "Body Area Network and Its Standardization at IEEE 802.15.BAN".

[4] J.-I. Takada and T. Aoyagi, "Static Propagation and Channel Models in Body Area", (2008) October, pp. 6-8.

[5] J. Malhotra, "Performance Analysis of Diversity Combining Multichannel Receivers in Generic-Gamma Fading Channels", Tamkang Journal of Science and Engineering, vol. 14, no. 4, (2011), pp. 333-340.

[6] J. Hagedorn, J. Terrill, W. Yang, K. Sayrafian, K. Yazdandoost and R. Kohno, "A Statistical Path Loss Model for MICS”, IEEE 802.15-08-0519-01-0006, (2008) September.

[7] V. Kaur and J. Malhotra, "Performance Evaluation of M-ary Modulations through WBAN Channel IMACST", vol. 2.

[8] J. Hagedorn, J. Terrill, W. Yang, K. Sayrafian, K. Yazdandoost and R. Kohno, "MICS Channel Characteristics; Preliminary Results", IEEE 802.15-08-0351-00-0006, (2008) September.

[9] J. Hagedorn, J. Terrill, W. Yang, K. Sayrafian, K. Yazdandoost and R. Kohno, "A Statistical Path Loss Model for MICS", IEEE 802.15-08-0519-01-0006, (2008) September.

[10] O. Diallo, "Real -time query processing optimisation for cloud-based wireless body area network", Information Sciences, vol. 284, (2014), pp. 84-94.

[11] J. Kim, H. Soo Lee, J. Ki Pack and T. Hong Kim, "Channel modeling for medical implanted communication systems by numerical simulation and measurement", IEEE 802.15-08-0274-02-0006, (2008) May.

[12] K. Yekeh Yazdandoost and R. Kohno, “Antenna for Medical Implanted Communications System”, IEEE 802.15-07-0785-00-0ban, (2007) July.

[13] T. Aoyagi, J.-ichi Takada, K. Takizawa, N. Katayama, T. Kobayashi, K. Yekeh Yazdandoost, H.-bang Li and R. Kohno, "Channel model for wearable and implantable WBANs", IEEE 802.15-08-0416-04-0006, (2008) November.

[14] K. Karthika and A. Padmabeaula, "Performance Analysis of PSSK Modulation Scheme for High-Data Rate Implantable Medical Devices", IJESIT, vol. 2, no. 4, (2013) July.

[15] T. Aoyagi, "Channel Models for WBANs - NICT", doc: IEEE P802.15-08-0416-04-0006, (2008) November.

\section{Authors}

Er. Sukhraj Kaur received the B.Tech. degree in Electronics and Communication from Guru Nanak Dev University, Regional Campus, Jalandhar, India in 2014 and is pursuing M.Tech. in ECE(specialization in Communication Systems) from GNDU, Regional Campus, Jalandhar, India. Her research area includes Wireless Body Area Networks. She published 4 research paper in international journals so far.

Dr. Jyoteesh Malhotra B.Eng. M.Tech. . PhD. Is involved in teaching and research at Electronics and Communication department, Guru Nanak Dev University, Regional Campus, Jalandhar, India. His research areas of interest include Statistical modeling of Fading Channels, Fading mitigation techniques in Wireless Communication and Optimization of WBAN air interface. Dr. Malhotra has more than 90 research publications and authored 02 books. He is a life member of I.S.T.E. and editorial board member of many International Journals of repute. 
International Journal of Advanced Science and Technology Vol.85 (2015) 